\title{
Eficacia del sulfato ferroso y multimicronutrientes en el incremento de hemoglobina en los niños de 6 a 36 meses de edad, en los distritos de San Juan de Rontoy y Llamellín, provincia Antonio Raimondi, Ancash
}

\author{
Neisi Becerril-Grandez ${ }^{1 *}$ y Julio Mendigure-Fernández ${ }^{1}$ \\ ${ }^{\prime}$ E.A.P de Enfermería, Facultad de Ciencias de la Salud, Universidad Peruana Unión, Alt. Km 19; Carretera Central, Chosica, Lima
}

\begin{abstract}
RESUMEN
Objetivo: Determinar la eficacia del sulfato ferroso y multimicronutrientes en el incremento de la hemoglobina en los niños de 6 a 36 meses de edad, en los distritos de Llamellín y San Juan de Rontoy en la provincia de Antonio Raimondi, Ancash. Material y Métodos: Utilizando el diseño preexperimental, se seleccionaron en total 100 niños; 55 del distrito de Llamellín, de los cuales el 50.9\% fueron mujeres y 49.1\% varones. Del distrito de San Juan de Rontoy fueron seleccionados 45 niños, 55.6\% fueron mujeres y 44.4\% varones. En ambos distritos los niños con anemia moderada o severa, recibieron suplemento de ferrán $1 \mathrm{mg} / \mathrm{kg} /$ día; en tanto que los niños con anemia leve o sin anemia recibieron suplemento de multimicronutrientes 1 sobrecito diario, conocido como "chispitas". Resultados: Al inicio del programa el 3.6\% tenía anemia severa, 50.9\% anemia moderada, 16.4\% anemia leve y el $29.1 \%$ estuvo libre de anemia; es decir, que en global el 70.9\% de los niños de Llamellín tenía anemia. En el distrito de San Juan de Rontoy, el 6.7\% tenía anemia severa, 26.7\% anemia moderada, 33.3\% anemia leve y el 33.3\% sin anemia; por tanto, el $66.7 \%$ presenta algún grado de anemia. Durante la intervención la media de la hemoglobina entre los niños de Llamellín que recibieron ferrón, se incrementó de 8.1 a $10.7 \mathrm{gr} / \mathrm{dl}(\mathrm{p}<0.05)$. Asimismo, en el distrito de San Juan de Rontoy la media de la hemoglobina se incrementó de 8.9 a $10.7 \mathrm{gr} / \mathrm{dl}(\mathrm{p}<0.05)$. En tanto que la administración de multimicronutrientes, en el distrito de Llamellín, tuvo como resultado el incremento discreto de hemoglobina de 11.0 a $11.3 \mathrm{gr} / \mathrm{dl}(\mathrm{p}>0.05)$. Asimismo, en el distrito de San Juan de Rontoy se incrementó ligeramente de 11.1 a $11.6 \mathrm{gr} / \mathrm{dl}$ ( $\mathrm{p}>0.05)$. Conclusiones: La administración del Ferrán en niños de los distritos de Llamellín y San Juan de Rontoy en forma de suplemento es eficaz para revertir la anemia ferropénica en los niños de 6 a 36 meses de edad $(\mathrm{p}<0.05)$.
\end{abstract}

Palabras clave: Anemia, hemoglobina, sulfato ferroso, multimicronutrientes.

\begin{abstract}
Objective: To determine the efficacy of ferrous sulfate and multimicronutrient in increasing hemoglobin in children 6 to 36 months of age, in the districts of San Juan de Llamellín and Rontoy in Antonio Raimondi province, Ancash. Material and Methods: Using the pre experimental design, we selected a total of 100 children, 55 Llamellín district, of which $50.9 \%$ were women and $49.1 \%$ men. District of San Juan de Rontoy were selected 45 children, 55.6\% were female and $44.4 \%$ male. In both districts, children with moderate or severe anemia, ferran supplement received $1 \mathrm{mg} / \mathrm{kg} / \mathrm{day}$, while children with mild anemia or without anemia received supplemental multimicronutrient 1 packet daily, known as "sprinkles". Results: At the beginning of the program to $3.6 \%$ had severe anemia, $50.9 \%$ moderate anemia, mild anemia $16.4 \%$ and $29.1 \%$ were free of anemia, bone, that overall $70.9 \%$ of children were anemic Llamellín. In the district of San Juan de Rontoy, 6.7\% had severe anemia, 26.7\% moderate anemia, mild anemia $33.3 \%$ and $33.3 \%$ without anemia, therefore, $66.7 \%$ have some degree of anemia. During the intervention, the mean hemoglobin among children who received ferran Llamellín was increased from 8.1 to $10.7 \mathrm{~g} / \mathrm{dl}$ (p $<0.05)$. Also in the district of San Juan de Rontoy mean hemoglobin increased from 8.9 to $10.7 \mathrm{~g} / \mathrm{dl}(\mathrm{p}<0.05)$. While multimicronutrient administration in the district resulted Llamellín, increased hemoglobin discrete 11.0 to $11.3 \mathrm{~g} / \mathrm{dl}(\mathrm{p}>0.05)$. Also in the district of San Juan de Rontoy increased slightly from 11.1 to $11.6 \mathrm{~g} / \mathrm{dl}(\mathrm{p}>0.05)$. Conclusion: The administration of ferran in children Llamellín districts and San Juan de Rontoy in supplement form is effective in reversing iron deficiency anemia in children $6-36$ months of age $(p<0.05)$.
\end{abstract}

Keywords: Anemia, hemoglobin, ferrous sulfate, multimicronutrient.

*Correspondencia: neiberg8@hotmail.com, 51-1-942490974 


\section{INTRODUCCIÓN}

La anemia es un síndrome caracterizado por la disminución en la cifra de hemoglobina o de los eritrocitos por debajo de los niveles considerados normal determinada edad, sexo y altura sobre el nivel del mar (OMS 2011).

La deficiencia de hierro sigue siendo lo más común de deficiencia nutricio-prevenibles pese a los objetivos globales para su reducción.

La OMS (2011) estima que en el mundo existen aproximadamente 2000 millones de personas anémicas y que cerca del $50 \%$ de los casos pueden atribuirse a la carencia de hierro. Los grupos etarios que presentan las más altas prevalencias son los lactantes y preescolares, porque se encuentran en un período de crecimiento y desarrollo rápido. En los países en desarrollo, el déficit de hierro se observa en una proporción del $40 \%$ al $60 \%$ de los niños.

En Latinoamérica el estado de ferropenia crónica y anemia manifiesta que afecta al $52.55 \%$ de la población, siendo más grave el problema en la infancia. Las evidencias señalan que las madres son las que condicionan los hábitos y patrones de alimentación del niño influyendo en su estado nutricional, el que a su vez condiciona su potencial de desarrollo y crecimiento (Wagner, 2004).

La OMS ha establecido entre sus objetivos prioritarios, la reducción o eliminación de esta carencia mediante estrategias como la educación nutricional de la población, la suplementación con hierro y el enriquecimiento de los alimentos tal como lo menciona (Antunes \& Costa, 2002).

En el Perú, la anemia muestra una tendencia decreciente en los últimos años. En el año 2011, aproximadamente, 734 mil niños y niñas menores de tres años tenían anemia, representando el $41.6 \%$ de este grupo de edad 46.6\%. Según Wagner (2004), se admite que cualquier niño puede padecer anemia por déficit de hierro, sin embargo, los sectores de mayor riesgo son: los niños menores de dos años, ya que en este período el crecimiento es muy acelerado y al mismo tiempo la alimentación que suelen recibir no les aporta la cantidad de hierro que necesitan. En los primeros doce meses de vida el bebé triplica su peso corporal y los depósitos de hierro que ha formado durante la gestación le alcanzan solo hasta aproximadamente los cuatro meses. Aparte que, a medida que va creciendo el niño, ciertos parásitos intestinales los cuales son tan frecuentes, suelen provocar anemia.

En el mundo se emplean diversas estrategias para enfrentar las deficiencias de micronutrientes: tales como la diversificación alimentaria, la fortificación de alimentos, la suplementación medicamentosa, la biofortificación y la fortificación casera llamada también suplementación múltiple y otros. (Huamán, 2010).

Licata (2012) refiere que los niños nacen con reservas de hierro suficientes para cubrir sus demandas nutricionales entre los 4 a 6 meses. El inicio de la alimentación complementaria, a partir del sexto mes, es de suma importancia porque las reservas de hierro están agotándose y el crecimiento del niño continúa, si estas no son cubiertas lo suficiente. Lamentablemente muchos niños antes de cumplir el año terminarán con anemia.

Según la OMS y el Instituto Nacional de Estadística e Informática (INEI, 2009), el 37.2\% de niñas y niños menores de cinco años padece de anemia, proporción menor a la observada en el año 2002 en el cual era un $49.6 \%$; sin embargo, la cifra resulta mayor cuando revisamos las prevalencias según grupo etario. Afecta al $75.2 \%$ de niñas y niños de 6 a 8 meses y alcanza el $72 \%$ en niñas y niños de 9 a 11 meses de edad, siendo aún elevada en niñas y niños de 12 a 17 meses de edad $60.3 \%$, mientras que en los infantes de 18 a 59 meses los porcentajes son menores.

La OPS (2008) señala que las deficiencias de vitaminas y minerales, en particular las de hierro, vitamina A y zinc, afectan a más de 2000 millones de personas en todo el mundo. Los niños pequeños son muy vulnerables debido al rápido crecimiento y a prácticas dietéticas inadecuadas.

También hay evidencias que indican que el porcentaje de anemia es mayor en niñas y niños de madres con primaria y sin educación $42.7 \%$ y $41.9 \%$ respectivamente; por sexo, los niños tienen mayor prevalencia de anemia que las niñas 38.3 y $36 \%$ respectivamente. La anemia es más frecuente entre niñas y niños residentes del área rural $44.1 \%$ de la sierra $46.6 \%$.

De acuerdo a los bancos de datos del Programa Mundial de Alimentos (PMA, 2008) en Perú más del $50 \%$ de niños y niñas menores de 5 años tiene anemia. La prevalencia de anemia en niños menores de 5 años en la región Lima es $58.45 \%$, a nivel distrital estos porcentajes son mayores (CENAN-2005).

El INEI (2010) reporta indicadores de resultados de los programas estratégicos 2010, que tienen una comparación de cuatro años en lo que respecta en niños de 6 a 36 meses con anemia, teniendo el resultado siguiente: año 2000 el $60.9 \%$ presentó anemia, 2007 el $56.8 \%$ tuvo anemia, en el 2009 un $50.4 \%$ estuvo 
con anemia y 2010 el 50.3\% aún presentan anemia los niños en este grupo etario. Asimismo, la prevalencia de anemia en el Perú el año 2000 fue de $60.9 \%$, en el año 2007 fue de $56.8 \%$, en el año 2009 es de $50.4 \%$ y en el año 2011 es $46.6 \%$.

Estos promedios nacionales distan mucho de las cifras locales más específicas. Así, por ejemplo, en Huaraz se ha encontrado altas prevalencias de anemia. En el distrito de Haija la anemia llega a $42.4 \%$, en el distrito de Recuay hay un $45.7 \%$ de anemia, en el distrito de Ocres llega a $52.3 \%$ de anemia, en la provincia de Huari la prevalencia de anemia es de $49.92 \%$, en la provincia de Antonio Raimondi presenta un 62.38\%, como también en los distritos de Llamellín y San Juan de Rontoy donde la anemia en niños menores de 3 años tiene una prevalencia de $68.9 \%$ (ADRA, 2010).

Ante este panorama, en el Perú, el Ministerio de Salud (MINSA), como autoridad sanitaria nacional, mediante la Dirección General de Salud de las personas a través de subprogramas de Crecimiento y Desarrollo del Niño (CRED) y el Programa Nacional de Prevención y Deficiencia de Micronutrientes (PREDEMI) del MINSA, desarrollaron estrategias en conjunto para prevenir la deficiencia de hierro, mediante la suplementación del sulfato ferroso, como profilaxis a niños mayores de 6 meses.

Por otro lado, existen instituciones no gubernamentales que toman mucho interés en la salud de los niños en las zonas andinas del Perú, dentro de ellos tenemos a la Agencia Adventista de Desarrollo y Recursos Asistenciales (ADRA) que se encuentra interviniendo en la región Ancash y es la primera organización en introducir el suplemento de los multimicronutrientes. Hay estudios que indican que la suplementación de multimicronutrientes puede disminuir la anemia.

Según el Arce (2010) menciona que la intervención con suplementos de multimicronutrientes y acciones preventivas benefició aproximadamente a 109,496 niñas y niños menores de tres años de los departamentos de Apurímac, Ayacucho y Huancavelica. Considerada una alternativa novedosa, ya que su efectividad y aceptabilidad ha sido probada en múltiples ensayos clínicos y comunitarios de diversos países, como en el altiplano boliviano donde se demostró una tasa de respuesta de $91 \%$, después de 60 días de suplementación diaria.

Los multimicronutrientes, o también llamadas chispitas, son una bolsita con un contenido de polvo que contiene una mezcla básica de hierro microencapsulado, zinc, vitamina. A, vitamina $\mathrm{C}$ y ácido fólico para mejorar el estado nutricional que se puede se polvorear sobre cualquier alimento semisólido.
Habiendo visto la problemática y recorrido los distritos de Llamellín y San Juan de Rontoy en la provincia Antonio Raimondi, se recogió la carencia de los habitantes de esta provincia, ya que los niños sufren de enfermedades por el difícil acceso a los servicios de salud adecuados, problemas de saneamiento, bajos ingresos y alimentación inadecuada, poca productividad de alimentos ricos en hierro, costumbres y grado de instrucción de las madres.

También se pudo ver claramente que los alimentos que consumen los niños carecen de nutrientes. Se observa un limitado consumo de menestras, frutas y verduras, relacionado con las condiciones adversas del clima y la altura que no favorece la digestión. Las anemias en niños forman parte del círculo vicioso de las enfermedades prevalentes, asociadas a las infecciones parasitarias, enfermedades diarreicas, infecciones respiratorias y una alimentación deficiente, con resultados distritales reflejando que las condiciones de los niños no son diferentes al promedio nacional respecto a la anemia en niños.

El objetivo del estudio es determinar la eficacia del sulfato ferroso y multimicronutrientes en el incremento de la hemoglobina en los niños de 6 a 36 meses de edad, en los distritos de Llamellín y San Juan de Rontoy en la provincia de Antonio Raimondi, Ancash.

\section{MATERIAL Y MÉTODOS}

La investigación se trata de un estudio preexperimental porque se hicieron mediciones del nivel de hemoglobina en sangre antes y después de la administración del tratamiento en un solo grupo de niños, consistente en aplicación de sulfato ferroso y multimicronutrientes, (chispitas) en los niños de los distritos de San Juan de Rontoy y Llamellín provincia Antonio Raimondi, región Ancash.

La población estuvo conformada por 100 niños de 6 a 36 meses de edad, de los cuales 45 pertenecen al distrito San Juan de Rontoy y 55 niños al distrito de Llamellín; todos ellos fueron beneficiarios del proyecto Ally Micuy.

Para fines de la presente investigación, se inició con el dosaje de hemoglobina de los niños menores de 36 meses de ambos distritos, identificando a los niños con hemoglobina menor y mayor a $11 \mathrm{~mm} / \mathrm{dl}$ de sangre, quienes conformaron la población de estudio en los distritos de San Juan de Rontoy y Llamellín Provincia Antonio Raimondi, Ancash.

Para la recolección de datos se utilizó una ficha de control (tabla), creada específicamente para esta 
investigación, en los cuales se incluirán datos generales de la población en estudio.

Posteriormente se ingresó a un programa de sistema de monitoreo nutricional, en el cual se hizo el análisis comparativo de niveles de hemoglobina en los niños de la población de estudio.

El análisis y tabulación de los datos obtenidos se realizó por medio del paquete estadístico SPSS versión 15.0 para Windows y el programa de Excel.

\section{RESULTADOS}

La distribución de los participantes en el estudio según las variables de residencia y sexo, 28 niños del distrito de Llamellín son de sexo femenino que hace el 50.9\% y la diferencia $(49.1 \%)$ son del sexo masculino. En tanto que en el distrito de San Juan de Rontoy, de los 45 participantes $55.6 \%$ fueron del sexo femenino y el $44.4 \%$ fueron del sexo masculino. Al iniciar el estudio, se estableció el nivel de anemia de los niños menores de 6 a 36 meses de edad en los distritos de Llamellín y San Juan de Rontoy. En del distrito de Llamellín, el 3.6\% tenía anemia severa, 50.9\% anemia moderada, 16.4\% anemia leve y el $29.1 \%$ estuvieron libres de anemia; es decir, que en general el 70.9\% de los niños de Llamellín tenía anemia y el $29.1 \%$ tenía la hemoglobina dentro de los parámetros normales. En el distrito de San Juan de Rontoy, el $6.7 \%$ tenía anemia severa, $26.7 \%$ anemia moderada, $33.3 \%$ anemia leve y el $33.3 \%$ sin anemia; por tanto, el $66.7 \%$ presentan algún grado de anemia y el $33.3 \%$ están libre de anemia.

Tabla 1

Hemoglobina en los niños de 6 a 36 meses de edad antes y después de la administración de los multimicronutrientes en los distritos de Llamellín y San Juan de Rontoy, en la provincia de Antonio Raimondi Ancash 2011.

\begin{tabular}{cccccc}
\hline \multirow{2}{*}{ Distrito } & Tipo de & \multicolumn{2}{c}{ Antes del } & \multicolumn{2}{c}{ Después } \\
& Anemia & \multicolumn{2}{c}{ Tratamiento } & N tratamiento \\
& Sin anemia & 16 & $64 \%$ & 19 & $76 \%$ \\
\hline \multirow{2}{*}{ Llamellín } & Leve & 9 & $36 \%$ & 4 & $16 \%$ \\
& Moderada & - & - & 2 & $8 \%$ \\
Severa & 25 & $100 \%$ & 25 & $100 \%$ \\
San Juan de & Sin anemia & 15 & $50 \%$ & 21 & $72.4 \%$ \\
Rontoy & Leve & 15 & $50 \%$ & 5 & $17.2 \%$ \\
& Moderada & - & - & 3 & $10.3 \%$ \\
& Anemia Severa & 30 & $100 \%$ & 29 & $100 \%$ \\
\hline
\end{tabular}

En la tabla 1 se observa una comparación de la línea de base de la anemia antes y después del tratamiento con multimicronutrientes. En el distrito de Llamellín, los niños sin anemia se incrementaron en 12 puntos porcentuales, al pasar de $64 \%$ al $76 \%$. Los niños con anemia disminuyeron en el período de tratamiento de $36 \%$ al $16 \%$, es decir se experimentó un notable descenso de $20 \%$. Sin embargo, llama la atención que dos niños, al finalizar el tratamiento, en lugar de mantener el nivel de hemoglobina, inesperadamente terminaron con anemia moderada. Aun así, se puede observar que ha disminuido la anemia presumiblemente atribuida a la suplementación de micronutrientes. La media de la hemoglobina se incrementó ligeramente de 11.0 a $11.3 \mathrm{gr} / \mathrm{dl}$. Al efectuar el análisis mediante la prueba de $\mathrm{T}$ para muestras relacionadas, las variaciones de la hemoglobina antes y después del tratamiento con multimicronutrientes en el distrito de Llamellín, esta arrojó $p=0.143$; por tanto, no es significativo $(p>0.05)$

En el distrito de San Juan de Rontoy, de los 30 niños que fueron incluidos para el tratamiento con multimicronutrientes, el $50 \%$ estaba sin anemia al inicio del programa y esta condición se incrementó a $72.4 \%$ al finalizar el programa, es decir, un incremento de $22.4 \%$. Los niños con anemia leve que al inicio representaban el $50 \%$ de los que recibieron los multimicronutrientes estos disminuyeron a $17.2 \%$, lo que representa un descenso en 33 puntos porcentuales. Al igual que en el distrito de Llamellín, estos resultados podrían atribuirse presumiblemente a 
la suplementación con multimicronutrientes. la media de la hemoglobina se incrementó ligeramente de 11.1 a $11.6 \mathrm{gr} / \mathrm{dl}$. Al efectuar en análisis mediante la prueba de $\mathrm{T}$ para muestras relacionadas, las variaciones de la hemoglobina antes y después del tratamiento con multimicronutrientes en el distrito de San Juan de Rontoy, esta arrojó $p=0.2$; por tanto, este incremento resulta estadísticamente significativo $(p>0.05)$.

Tabla 2

Anemia en los niños de 6 meses a 36 meses de edad antes y después de la administración de sulfato ferroso en los distritos de Llamellín y San Juan de Rontoy en la provincia de Antonio Raimondi, Ancash 2011.

\begin{tabular}{|c|c|c|c|c|c|}
\hline \multirow{2}{*}{ Distrito } & \multirow{2}{*}{ Tipo de anemia } & \multicolumn{2}{|c|}{$\begin{array}{c}\text { Antes del } \\
\text { Tratamiento }\end{array}$} & \multicolumn{2}{|c|}{ Después del tratamiento } \\
\hline & & & & & $\%$ \\
\hline \multirow{3}{*}{ Llamellín } & Normal & 28 & $93.3 \%$ & 18 & $60.0 \%$ \\
\hline & Leve & 2 & $6.7 \%$ & 4 & $13.3 \%$ \\
\hline & Moderada & & & 8 & $26.7 \%$ \\
\hline \multirow{5}{*}{$\begin{array}{l}\text { San Juan de } \\
\text { Rontoy }\end{array}$} & Severa & 30 & $100 \%$ & 30 & $100 \%$ \\
\hline & Normal & 12 & $80.0 \%$ & 10 & $66.7 \%$ \\
\hline & Anemia Leve & & & 1 & $6.7 \%$ \\
\hline & $\begin{array}{r}\text { Anemia } \\
\text { Moderada }\end{array}$ & 3 & $20.0 \%$ & 4 & $26.7 \%$ \\
\hline & Anemia Severa & 15 & $100 \%$ & 15 & $100 \%$ \\
\hline
\end{tabular}

En el distrito de Llamellín, los niños sin anemia se incrementaron en 60 puntos porcentuales, al pasar de $0 \%$ al $60 \%$. Los niños con anemia moderada, disminuyeron en el período de tratamiento de $93.3 \%$ al $26.7 \%$, es decir, se experimentó un notable descenso de $66.6 \%$. Asimismo, los niños que tenían anemia severa disminuyeron de $6.7 \%$ al $0 \%$ es decir, todos los casos de anemia severa se revirtieron atribuido a la suplementación de Ferrán. Y la media de la hemoglobina entre los niños se incrementó ligeramente de 8.9 a $10.7 \mathrm{gr} / \mathrm{dl}$. Al efectuar en análisis mediante la prueba de T para muestras relacionadas, las variaciones de la hemoglobina antes y después del tratamiento con Ferrán en el distrito de Llamellín, esta arrojó $\mathrm{p}=0.00$; por tanto, este hallazgo resultó estadísticamente significativo $(p<0.05)$.

En el distrito de San Juan de Rontoy, de los 15 niños que fueron incluidos para el tratamiento con Ferrán, el $80 \%$ estaban con anemia moderada al inicio del programa y esta condición disminuyó a $26.7 \%$ al finalizar el programa, es decir el descenso representa 53 puntos porcentuales. Los niños con anemia severa que al inicio representaban el 20\% de los que recibieron Ferrán, estos no presentaron anemia severa al culminar el programa en ninguno de ellos. Al igual que en el distrito de Llamellín, estos resultados son atribuidos a la suplementación con Ferrán. Y la media de la hemoglobina se incrementó de 8.1 a $10.7 \mathrm{gr} / \mathrm{dl}$. Al efectuar el análisis mediante la prueba de $\mathrm{T}$ para muestras relacionadas, las variaciones de la hemoglobina antes y después del tratamiento con
Ferrán en el distrito de San Juan de Rontoy, esta arrojó $p=0.000$; por tanto, el hallazgo es estadísticamente significativo $(p<0.05)$.

\section{DISCUSIÓN}

Ancash es una región de la sierra peruana caracterizado por los elevados niveles de anemia infantil. Las estadísticas nacionales de INEI (2010), indican que en el distrito de Haija la anemia llega a $42.4 \%$, en Recuay a $45.7 \%$, en Ocres a $52.3 \%$, y en la provincia de Huari a 49.92\%. Asimismo, en los distritos de Llamellín y San Juan de Rontoy, el panorama de la anemia es alarmante. Por ejemplo, al inicio del estudio, la prevalencia de anemia en el distrito de Llamellín alcanzaba a $70.9 \%$ mientras que en San Juan de Rontoy era de $66.7 \%$. Como puede apreciarse, comparativamente con los otros distritos de la región, estas superan largamente el promedio regional que según el INEI (2010), que en Ancash es $54.1 \%$ y el promedio nacional para el ámbito rural en ese mismo año estaba en $56.6 \%$. Sin embargo, posterior a la intervención, la prevalencia de Anemia disminuyó en Llamellín a 32.7\% y en San Juan de Rontoy a $28.9 \%$, respectivamente, con lo cual queda demostrado la eficacia de la intervención con suplemento de sulfato ferroso de $1 \mathrm{mg} / \mathrm{kg} /$ día de Ferrán, durante el período de tres trimestres complementado con otras acciones de educación, información y comunicación, para combatir la anemia en los niños de 6 a 36 meses de edad. 
Este hallazgo, coincide con los resultados reportados por Morán T; Rodríguez R; Rojas C; Canchaya (2012) quienes indican la eficacia de la administración de suplemento de sulfato ferroso (Ferrán) para la reducción de la anemia en niños de 6 a 35 meses de edad, en comunidades rurales de Ancash, en el ámbito del proyecto Ally Micuy de Cáritas del Perú con el apoyo del Fondo Minero Antamina.

El hallazgo que reportamos, coincide también con los resultados obtenidos por Sotelo (2002), quien reporta la administración de sulfato ferroso de $3.5 \mathrm{mg} /$ $\mathrm{kg}$ /día en menores de tres años, resultó efectivo para incrementar los niveles de hemoglobina, así como también puede revertir cualquier grado de anemia ferropénica en niños que participaron en el estudio.

En el mismo sentido, Huamán. (2010) reportó la eficacia del sulfato ferroso para el tratamiento de la anemia ferropénica al término de los 90 días de suplementación con Ferrán, experimentándose valores normales para hemoglobina, hematocrito, volumen corpuscular medio, ferremia y saturación $(p<0,05)$, atribuyéndolo al sulfato ferroso como responsable del incremento más precoz y de mayor intensidad en la hemoglobina, ya que permite la normalización de valores en mayor cantidad de pacientes y presenta similar tolerabilidad y grado de adhesión al tratamiento que el hidróxido férrico polimaltosado.

Con relación a la suplementación con multimicronutrientes (chispitas) se administró un sobre diario a los niños que tenían anemia leve y a los niños que no presentaban anemia durante tres trimestres. En este estudio se encontró que no tuvo mayores consecuencias positivas para la diminución de la anemia en el distrito de San Juan de Rontoy donde la media de la hemoglobina aumento de 11.0 a $11.6 \mathrm{gr} / \mathrm{dl}$, mientras que en Llamellín se incrementó de 11.0 a $11.3 \mathrm{gr} / \mathrm{dl}$. Estos incrementos discretos no fueron suficientes para demostrar la eficacia en el combate de la anemia; lo cual coincide con los hallazgos de Huamán (2010), quienes a pesar de que se encontró una correlación positiva entre el número de sobres de Chispitas consumidos y los valores de hemoglobina (rho Spearman=0,10, $p=0,008)$; sin embargo, cuando se planteó dividir la categoría de 60 o más sobres consumidos (de 60 a 89 y de 90 a más sobres), no se encontró diferencias en las medias de hemoglobina ni en las prevalencias de anemia.

Es necesario hacer notar que en el Perú se realizó una intervención en zonas urbanas pobres de Chiclayo (costa norte) durante el año 2000 para mujeres en edad fértil y adolescentes, así como a menores de cinco años, con tabletas de multimicronutrientes una vez a la semana durante seis meses en dos períodos, esta intervención no encontró una mejora en el grupo intervenido respecto a su medición basal; sin embargo, el grupo no intervenido incrementó sus niveles de anemia, por lo que concluyeron que la intervención evitó el desarrollo de anemia según López de Romaña D, Verona S, Vivanco OA, Gross R. (2006).

Situación similar podría haber ocurrido en la región Apurímac, donde no se puede afirmar la inexistencia de un efecto positivo de la intervención dado que no tenemos los datos de un grupo control que tenga las mismas características de pobreza, ruralidad y altitud.

También, es preciso anotar que en este estudio se identifican desde nuestra perspectiva algunos factores que facilitaron su implementación, entre ellos: la fluida coordinación y participación de las autoridades comunales, de las madres beneficiarias, los agentes comunitarios de salud y disposición del personal responsables del área del niño de los puestos de salud que coadyuvaron al cumplimiento de las capacitaciones mediante las sesiones educativas y demostrativas, así como las visitas domiciliarias.

También identificamos algunos factores limitantes, como la dispersión de la población y la distancia geográfica entre las comunidades, la altitud, inicio de las cosechas agrícolas y el nivel educativo de la madre, lo cual hacía aún más difícil realizar un seguimiento minucioso a las familias participantes.

\section{CONCLUSIONES}

El nivel de anemia, al inicio del programa, en el distrito de Llamellín era el 70.9\% y en San Juan de Rontoy el $66.7 \%$, en los niños menores de 6 a 36 meses de edad

Ha quedado demostrada la eficacia del sulfato ferroso para revertir la anemia ferropénica en los niños de 6 a 36 meses de edad, en los distritos de Llamelín y San Juan de Rontoy $(p<0.05)$.

La administración de micronutrientes "Chispita" no es eficaz para disminuir los niveles de anemia ferropénica en los niños de 6 a 36 meses de edad, en los distritos de Llamellín y San Juan de Rontoy, a pesar que la media de la hemoglobina se incrementó discretamente entre los niños que participaron en el estudio. 


\section{REFERENCIAS BIBLIOGRÁFICAS}

Antunes, H., \& Costa, P. A. (2002). Prevalence of Deficiency Anemia According to Infant Nutrition. Porto: Faculty of Medicine.12-20.

Arce, M. (2010). Resultados de línea basal de intervención con multimicronutrientes en Apurímac. Instituto Nacional De Salud.

Huaman, L. (2010). Consumo de suplementos con multimicronutrientes chispitas ${ }^{\circledR}$ y anemia en niños de 6 a 35 meses: estudio transversal en el contexto de una intervención poblacional en Apurímac, Perú. Revista Peruana de Medicina Experimental y Salud Publica. Recuperado por http://www.rpmesins.gob.pe/index.php/rpmesp/ article/view/4225.

Instituto Nacional de Estadística e Informática (2009). Informe de indicadores de resultados de los programas estratégicos en salud, Perú.

Koontz, H., \& Weihrich, H. (2004). Administración una perspectiva global. México: McGraw-Hill Interamericana.

Lewis, S, Bain, J \& Bates, I. (2008). Hematológica práctica. (10a ed.). España. Elsevier.
Licata, M. (2012). El hierro en la nutrición. Revista de Nutrición, 11-12.

López de Romaña, D., \& Vivanco, G. (2006). Multimicronutrient supplementation against anemia among children, woman, and adolescent girls in loweincome areas of Chiclayo, Peru. Revista de Salud Pública Vol , 20-40.

Organización Panamericana de la Salud, (2008) Alimentación y Nutrición del niño pequeño: Memoria de la Reunión Subregional de los Países de Sudamérica.

Organización Mundial de la Salud. (2011). La anemia ferropénica evaluación, prevención y control: una guía para administradores de programas. Ginebra: Informe del Departamento de Nutrición para la Salud y el Desarrollo. Recuperado de http://www. who.int/vmnis/indicators/haemoglobin_es.pdf

Sotelo, N. (2002). Tratamiento de la deficiencia de hierro en preescolares. México: Gaceta Médica.

Wagner, P. (2004) La anemia: consideración fisiopatológicas, clínicas y terapéuticas (3ra edición). Working Group Latinoamérica: Lima. 